\title{
Reverse Engineering of 3D Models Based on Image Processing and 3D Scanning Techniques
}

\author{
A. Manor, A. Fischer \\ CMSR Laboratory for Computer Graphics and CAD \\ Department of Mechanical Engineering \\ Technion \\ Haifa, Israel 32000 \\ Email:meranath@tx.technion.ac.il
}

\begin{abstract}
D}$ laser scanners play an essential role in Reverse Engineering. Laser-based sensors are non-contact fast sensors and therefore are commonly used to digitize the object. However, 3D boundary detection of the 3D digitized points produced by laser-based scanners is problematic due to the fact that the sensor data is often unreliable near sharp edges.

A new method based on fusion of 3D scanned data and an intensity image is proposed to overcome the above problems and improve the accuracy of the reconstructed surface. The 3D scanned data provides 3D data explicitly, whereas an intensity image offers other 2D characteristic information such as texture and boundary. For smooth freeform surfaces that generate smooth 2D silhouettes, characteristic curves of the object such as boundaries can be derived from its image using edge-detection algorithms. The feasibility of the method is demonstrated by several examples.
\end{abstract}

Key words: Reverse engineering, image processing, surface reconstruction

\section{INTRODUCTION}

Currently Reverse Engineering (RE) plays an essential role in industrial CAE systems. With RE, objects can be digitized, reconstructed and represented as computerized models. Digitizing systems in RE are based on three main technologies: (a) coordinate measuring machines (CMMs), (b) 3D laser scanners, and (c) digital photogrammetry systems $[1,2,4,11]$. 
In CMMs, a contact measure device such as probe is used. Since this method is sufficiently robust and accurate, it is commonly used to sample points as part of the RE process $[12,15]$. However, its advantages are greatly diminished by the slow rate of data capture. In addition, the object's surfaces may be delicate or flexible and require non-contact measurement.

Laser-based range sensors are non-contact sensors and therefore are very fast [11]. The scanned data provides explicit 3D points from which a $3 \mathrm{D}$ model can be reconstructed. However, the 3D data generated by $3 \mathrm{D}$ laser scanners is not suitable for direct incorporation in CAD systems: the scanned data is irregular and scattered and requires intensive processing in order to reconstruct the surface of the object. Laser scanner systems provide an enormous amount of digitized point data, therefore requiring a timeconsuming data reduction process. In order to improve reconstruction processing, some scanned systems provide range image instead of scattered points. A sampled surface $z\left(x_{i}, y_{i}\right)$ is represented as a $2.5 \mathrm{D}$ image where each pixel $\left(x_{i}, y_{i}\right)$ contains the height. However, range sensors or CMMs are often not applicable, e.g. where objects are too big, too small, too far, immovable or hazardous.

Digital photogrammetry systems reconstruct 3D models from a set of 2D images. Using a triangulation technique, the range of points in the scene can be calculated by applying a matching process. This process, however, is time consuming and suffers from convergence problems. The information is not complete since some points are not "seen" in both images. Moreover, when multiple images are used, camera calibration is needed to correct for geometric errors and to account for the camera parameters.

Computer-vision techniques can be applied to range images alone to infer geometric and topological structure, but accuracy problems occur on the boundaries.

In this paper a new method is proposed which overcomes the above problems by approximating a surface using a 2D intensity image from a CCD camera and 3D laser-scanned points (Figure 1). The 3D scanned data provides 3D data explicitly, whereas an intensity image offers other 2D characteristic information such as texture and boundary. Fusion of 3D scanned data and an intensity image can highly improve the reconstruction process. When dealing with smooth freeform surfaces that generate smooth 2D silhouettes, characteristic curves of the object can be derived from its image using edge-detection algorithms. Furthermore, local surface orientation can be computed both from the boundary and from the interior surface. Another important surface feature-Gaussian curvature - can be derived from the 2D silhouette, since the sign of the Gaussian curvature of points on the 3D surface projecting onto the silhouette is the same as the sign 
of curvature of those projections. All the above image-inferred information can be used as constraints in the surface approximation stage.

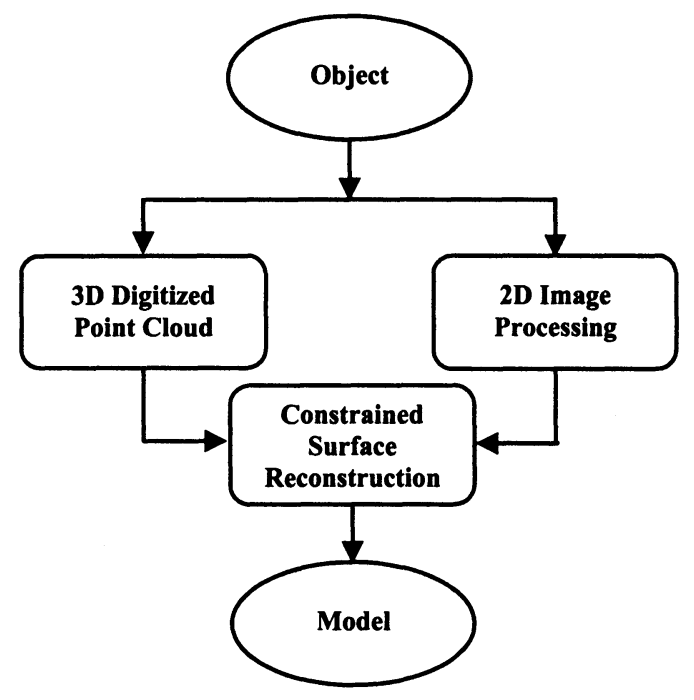

Figure 1: General block diagram of the proposed approach.

The paper focuses on extracting a 3D boundary, thus creating a basesurface parameterization from which the 3D surface is reconstructed. Section 2 describes the approach of surface fitting that is based on 3D approximation and image processing techniques. The algorithm that integrates the image processing technique with the LSQ approximation method is described in Section 3. The algorithm is demonstrated by several examples in Section 4. A summary and conclusions are presented in Section 5.

\section{APPROACH}

This paper focuses on surface reconstruction from 3D scattered data and 2D intensity image, while utilizing the surface boundary. The surface is represented by a B-Spline tensor surface. The stages of the proposed algorithm are as follows:

\section{Image Processing:}

1. Extracting the projected boundary from an intensity image using edge detection technique.

2. Representing the projected $2 \mathrm{D}$ discrete boundary as a continuous BSpline curve. 
3. Applying a matching technique on the set of $3 \mathrm{D}$ digitized points and the $2 \mathrm{D}$ curve. The matched $3 \mathrm{D}$ points will be recognized as boundary points.

\section{Surface Fitting:}

1. Approximating the $3 \mathrm{D}$ boundary points as a set of continuous $3 \mathrm{D}$ boundary curves.

2. Creating a 3D base surface where its boundary was computed in Stage 1 .

3. Assigning parameter values to the $3 \mathrm{D}$ digitized points according to the base surface.

4. Approximating the $3 \mathrm{D}$ digitized points as a B-Spline surface while interpolating the boundary curves.

The block diagram of the proposed algorithm is shown in Figure 1. Each of the above stages will be described in detail:

\subsection{Image Processing}

\subsubsection{Geometry image formation}

We distinguish between an intensity image, a grid of intensity values, and a range image, a grid of range values. In this paper we focus only on intensity images. There are many ways for directly obtaining a digital image of light intensity, such as vidicon and solid devices. Solid camera devices, such as charge-coupled device (CCD) cameras, are used widely since they are compact, have no geometrical distortions and can be easily mounted in a variety of places. The light intensity induces charges on the capacitors. The charges are read to a frame memory for each scan.

There are two parts to the [intensity] image formation process:

1. The geometry of image formation determines where the projection of a point in the scene will be located in the image plane.

2. The physics of light determines the brightness of a point in the image plane as a function of scene illumination and surface properties.

Since this research involves calibration of $3 \mathrm{D}$ world coordinates and 2D image coordinates, the geometry of image formation is highly important. The basic model for the projection of points in the scene onto the image plane is the perspective projection model.

\subsubsection{Edge point detection}

One of the most important image features in 3D computer vision is the 'edge' or the boundary line of a uniform region. There are four basic ways in 
which contours can arise in an image: (1) discontinuities in distance from the viewer; (2) discontinuities in surface orientation; (3) changes in surface reflectance; (4) illumination effects such as shadows, light sources and highlights. An edge in an intensity image may be defined as a discontinuity or abrupt change in the gray level (or color). In a range image, an edge can be recognized as a discontinuity in the depth value.

There are two common ways to extract edges from an image: (1) the edge method by which edge points are extracted and then linked into edges; (2) the region method, by which uniform intensity regions are computed and the boundaries of those regions are then extracted.

Edge points are detected in two steps: an edge operator is applied to every pixel to evaluate its likelihood as an edge point; then, edge points are determined on the basis of output values of the operator.

Edge detection in range images produced by laser-based scanners is problematic because the sensor data is often unreliable near sharp edges. The main effect is known as the "mixed point" problem and is illustrated in Figure 2, in which the laser footprint crosses the edge between two objects that are far from each other (or between an object and the background). In that case, the returned range value is some combination of the range of the two objects but does not have any physical meaning. This problem makes the accurate detection of occluding edges more difficult.

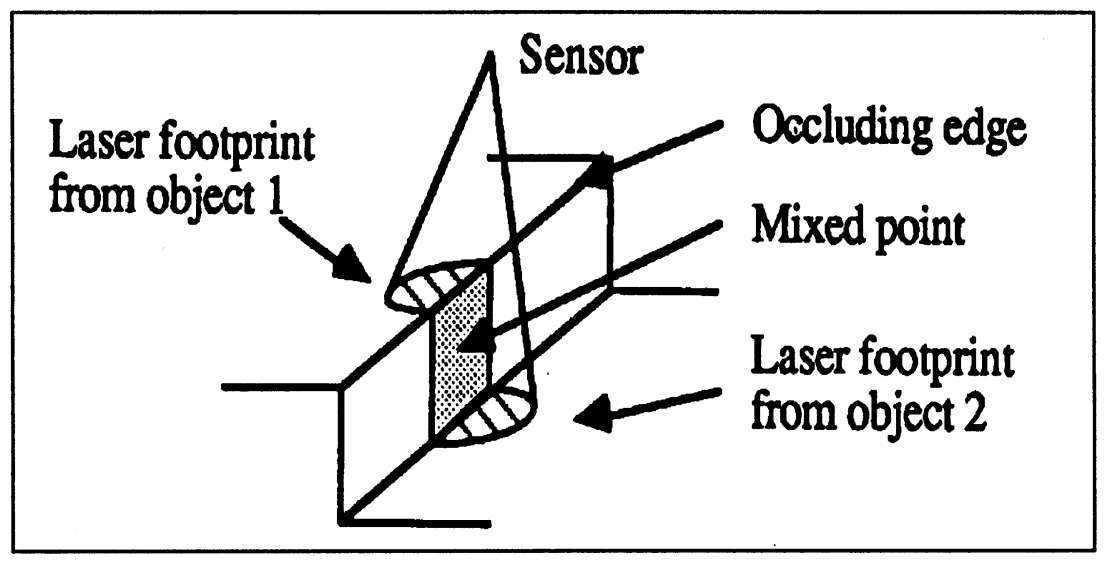

Figure 2: Edge measurement error in range data (from [5]).

To remove the effect of outliers and data-dropouts in range images, the data must be smoothed and low-pass filtered. The amount of noise decides the degree of smoothing to be performed on the data. However, smoothing tends to blur discontinuities in the range data, leading to a loss of geometric information, particularly at the boundary. 


\subsubsection{Boundary extraction}

An occluding contour is a contour that marks a discontinuity in depth; it usually corresponds to the silhouette of an object as seen in 2D projection. The two dimensional contour of the image of an object yields information about the three-dimensional shape [9].

Thus, it is desirable to aggregate the edge elements that constitute the boundary of an object. One possible form of aggregation results in a piecewise linear approximation-polygonal approximation-and can be obtained in two main ways [8]. One of these methods relies on the determination of $n$ critical points along the contour, which then define a polygon. The second relates to the use of successive approximations to iterate to a best-fit polygonal representation.

For smooth boundary representations, curve fitting methods are used. Parametric curves such as B-splines are suitable for representing continuous lines specified by a sequence of points.

\subsection{D Surface Reconstruction}

\subsubsection{B-Splines representation}

B-Splines curves and surfaces have wide applications in image processing and computer aided geometric design.

B-spline curves and surfaces are defined by the following equations:

$$
\begin{aligned}
& C(u)=\sum_{i=1}^{n_{u}} B_{u i}(u) \cdot \bar{V}_{i} \\
& S(u, v)=\sum_{i=1}^{n_{u}} \sum_{j=1}^{n_{v}} B_{u i}(u) \cdot B_{v j}(v) \cdot \bar{V}_{i j}
\end{aligned}
$$

where $u$ and $v$ are location parameters which uniquely locate a point on the curve or surface, and $\left\{B_{u i}(u)\right\},\left\{B_{v j}(v)\right\}$ are normalized B-splines.

A B-spline surface is completely defined by its orders $k_{u}$ and $k_{v}$, an ordered set of $n=n_{u} \times n_{v}$ control points (vertices) $\left\{\bar{V}_{i j}\right\}$, and two knot vectors $\xi=\left\{\xi_{i} / i=1 . . n_{u}+k_{u}\right\}$ and $\eta=\left\{\eta_{j} / j=1 . . n_{v}+k_{v}\right\}$.

\subsubsection{Parameterization}

A distinction is made between parameterization of digitized points and parameterization of knot vectors. When parametric values of the measured 
points are fixed, there should be an optimal set of corresponding knots for a fixed order and number of control points. On the other hand, if the knots are fixed first, there should also be a set of optimal location parameters for the measured points that yield optimal fitting results. Selection of an appropriate parameterization is essential for obtaining good surface fitting or for approximating results. Different parameterization strategies can be used to assign the location parameters: equally spaced, chordal, centripetal, geometric and affinity invariant parameters [6]. These methods are based on the assumption that the points are arranged in a grid and are therefore not suitable for irregularly spaced measured points. In such cases, methods based on the concept of projecting all points on a base surface (in the simple case - a parameter plane) can be used $[6,7]$.

\section{Parameterization using Base-Surface Projection}

Instead of trying to find an optimal parameterization, Kruth \& Ma [7] propose base surface parameterization. The suggested strategy is to project the digitized points onto a base surface which is a first approximation of the surface and to associate the projected points parameters with the corresponding sampled point. In contrast to other methods, the base surface is not necessarily a plane.

\subsubsection{Surface Reconstruction}

Surface reconstruction methods can be categorized into linear methods and curved surface fitting methods.

Linear Reconstruction: Digitized points are sampled as a cloud of points or scattered data. A volumetric object is usually constructed from scattered data by first building a triangular mesh. Then, several global and local criteria can be used to create the optimal triangular mesh [6].

Curved Surface Fitting Methods: Surfaces are usually reconstructed by interpolation or approximation methods [6]. When data points are obtained empirically by measurements (as in RE), approximation is more desirable than interpolation for overcoming noise errors and reducing computational complexity. The surface is fitted according to constant tolerance criteria or according to weights if the measurements have different accuracies [6]. For Cartesian and parametric surfaces, fitting methods are based on least square (LSQ) [5] and linear programming methods. The method described in [13] is an adaptive method, whereby cubic triangular Bezier surfaces are fitted to scattered dense data. LSQ approximation of data points with algebraic patches might produce surfaces with extraneous parts. 
There are two stages in reconstructing parametric surfaces: (1) parameterization of the surface, and (2) approximation of the surface.

The projected boundary can be approximated through several methods, such as B-spline approximation. Boundary space curves produce a type of four-sided patch tensor-product or a three-sided patch [3]. Next, a grid is defined on the parameter plane. The parameter values of any point can be calculated according to the grid.

Surface fitting methods must all handle the problem of inferring the correct surface topology. One of the most common methods is the least square fitting of B-spline surface [14]. This method is applied by adaptive global approximation algorithms using the least square method for B-spline.

\subsubsection{LSQ approximation of B-spline curves and surfaces}

In the approximation of a point set by a B-spline curve/surface, our aim is to solve the following problem: given $m$ not necessarily uniformly spaced points $P_{i}$ and associated parameter values $\left(u_{s}\right) /\left(u_{s}, v_{s}\right) /, s=0 . . m$, find an approximating B-spline curve/surface with respect to some prescribed error norm, i.e.: a set of control vertices $\left\{\bar{V}_{i j}\right\}$ is to be found.

For a B-spline curve written in parametric form as in (1), substituting the parameter values leads to a linear system of equations:

$$
\bar{P}_{s}=\sum_{i=1}^{n_{u}} B_{u i}\left(u_{s}\right) \bar{V}_{i} \quad s=0 . . m \text {. }
$$

In the case of a tensor-product B-spline surface written in parametric form as in (2), the system of linear equations is:

$$
\bar{P}_{s}=\sum_{i=1}^{n_{u}} \sum_{j=1}^{n_{v}} B_{u i}\left(u_{s}\right) B_{v j}\left(v_{s}\right) \bar{V}_{i j}, \quad s=0 . . m .
$$

A least squares fitting solution to equations (3) and (4) can be achieved when $m>n$.

\section{Constraint LSQ Fitting of B-spline Surfaces}

When some of the control vertices in the surface control mesh are set, the number of linear equations in set (4) is reduced, i.e. the degree of freedom for the least-squares fit is reduced. However, owing to the local support of the B-spline basis, this constraint is confined to only a few elements. 


\section{ALGORITHM DESCRIPTION}

The three major steps in the proposed algorithm are:

1. 3D boundary approximation

2. Parameterization of the scattered points using a base surface

3. Constrained LSQ surface fitting

\subsection{D boundary reconstruction from image}

Since the 3D boundary of the object is to be used as a constraint in the surface approximation, the first stage in the algorithm integrates data from both the intensity image and the range image to derive a $3 \mathrm{D}$ approximation of the boundary. This stage is broken down to smaller tasks:

(a) Extracting the discrete $2 D$ boundary from the intensity image

This extraction can be accomplished using threshold technique.

(b) Approximating the $2 D$ discrete digital curve to a continuous one

From the discrete representation of the 2D boundary we derive a continuous B-spline curve representation according to [10]. The B-Spline approximating the boundary of the object is superimposed on the intensity image of the object to be modeled.

\section{(c) Computing the perspective transformation}

In the experimental environment, the $\mathrm{CCD}$ camera and the $3 \mathrm{D}$ laser scanner are not placed on the same platform. Thus, we cannot assume that the range data and the intensity image are calibrated. Furthermore, the camera parameters (both intrinsic and extrinsic) are not known. Since accuracy according to ground truth is not needed, a technique that involves computing a perspective transformation matrix was chosen. The procedure requires specification of several correspondence pairs-3D coordinates and 2D image coordinates of a number of points of the object-to compute the perspective transformation matrix between the $3 \mathrm{D}$ and the $2 \mathrm{D}$ coordinates. This is done interactively, as shown in Figure 3. 


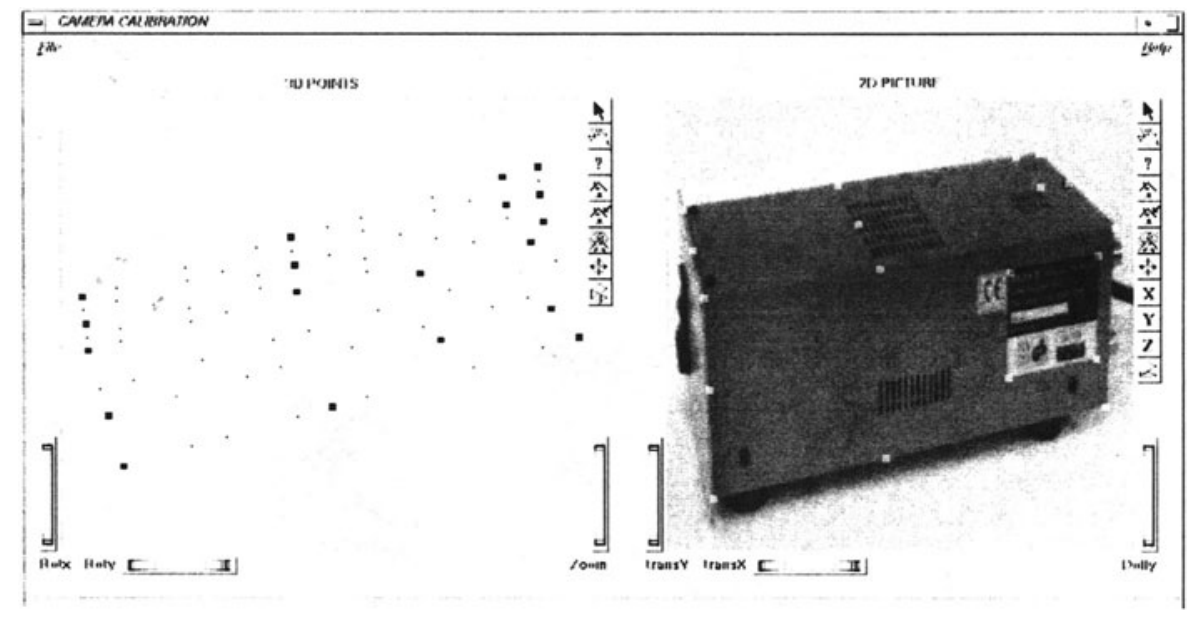

Figure 3: Interactive specification of correspondence pairs between 3D digitized points and a $2 \mathrm{D}$ image of an object (a camera adaptor).

Given the 3D world coordinates of a number of points and the corresponding 2D image coordinates, the coefficients in the perspective transformation matrix can be solved by least square solution of an overdetermined system of linear equations. Although this procedure is not exactly a camera calibration procedure as the camera parameters are still unknown, a reasonably good fit between the digitized points projection and the image was achieved (as in Figure 4).

\section{(d) Matching between projected $3 D$ digitized points and the $2 D$ curve}

The 3D range data is given as a cloud of scattered points. A matching technique is needed to find the digitized 3D points corresponding to the $2 \mathrm{D}$ boundary extracted from the image. The matching technique is as follows: For each 3D sample point

1. project on the image plane according to the computed perspective transformation;

2. determine if the projected point lies near the $2 \mathrm{D}$ curve using a distance tolerance criteria;

3. if so-mark the 3D sample point as a boundary point, and assign it the parameter of its projection on the curve.

As output from this step, we get an ordered list of 3D boundary points, where each point is assigned a parameter value. 


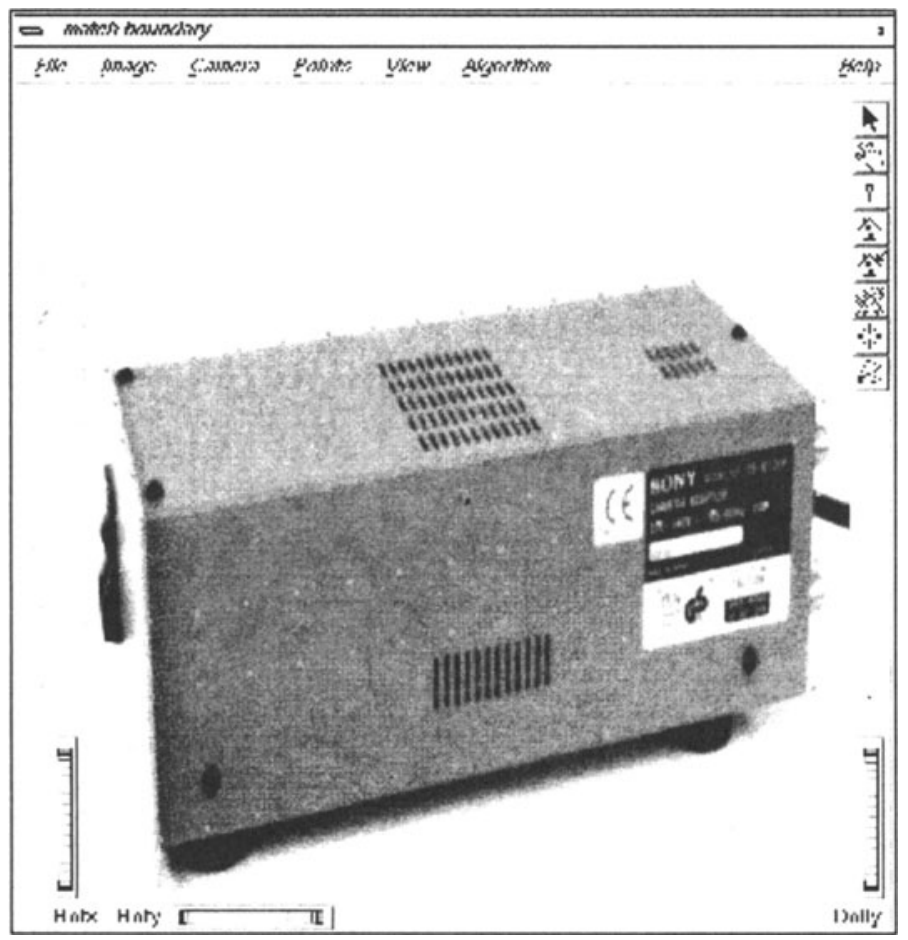

Figure 4: Projection of the 3D digitized points on the 2D image according to the perspective transformation computed for the correspondence pairs.

(e) Approximating the continuous $3 D$ boundary of the object

The goal in this step is to approximate the $3 \mathrm{D}$ contour of the object using four boundary curves, each being a B-spline curve approximating/ interpolating a partial set of the matched 3D boundary points.

The list of points and the corresponding parameter values from the previous step is input to a standard B-spline curve fitting procedure, and a single B-spline of the required order approximating the points is derived.

At this stage, the user interactively partitioned the 3D boundary curve into four curves by specifying the parameters of the four corner points.

\subsection{Parameterization of the sampled points using a base surface}

We use Kruth's approach to assign parameter values to randomly measured points for least squares fitting of B-spline surfaces [7]. The parameterization is realized by projecting the measured points to a base 
surface. The parameters of the projected points are then used as the parameters of the measured points. The base surface is in fact a first approximation of the final fitted surface, and in general it can be defined from four approximating boundaries. A Boolean-sum surface interpolating the four boundaries is defined in order to create a base surface in B-Spline form from its boundaries.

\subsection{Constrained LSQ surface fitting}

Once the scattered measured points have been parameterized and the 3D boundary of the object has been determined, we can proceed to fit a surface interpolating the boundary and approximating the inner measured data points.

Instead of computing all the coefficients (or control vertices) of the BSpline surface, we constrain the solution such that the boundary of the surface is fixed to some curve boundaries already created from the data. This is achieved by using the knowledge of the control points of the boundary BSplines (from the previous stage) that will form the surface boundary and removing them from the solution mechanism of the surface [16].

The boundary conditions are imposed by modifying the system of equations. The vertices of the control mesh at the boundaries are positioned so that the surface boundary is fixed to the pre-determined boundary curves. Hence, these vertices have to be isolated from the basis functions, i.e. the degree of freedom for the least-squares fit is reduced. This can be mathematically shown as follows:

The required surface is a B-spline surface of the form:

$$
S(u, v)=\sum_{i=1}^{n} \sum_{j=1}^{m} B_{u i}(u) \cdot B_{v j}(v) \cdot \bar{V}_{i j},
$$

in which case a boundary (e.g. at $v=v_{0}=0$ ) is represented as:

$$
b_{u 1}=S\left(u, v_{0}\right)=\sum_{i=1}^{n_{u}}\left[\sum_{j=1}^{n_{v}} B_{v j}\left(v_{0}\right) \cdot \bar{V}_{i j}\right] \cdot B_{u i}(u)
$$

For this surface boundary to coincide with a prescribed boundary curve, $C(u)$ :

$$
C(u)=\sum_{i=1}^{n_{u}} \bar{W}_{i} \cdot \bar{B}_{i}(u)=b_{u 1}
$$

the following equality has to hold (for $i=1 . . n_{u}$ ):

$$
\bar{W}_{i}=\sum_{j=1}^{n_{v}} B_{v j}\left(v_{0}\right) \cdot \bar{V}_{i j}
$$


To isolate the boundary vertices, we set the multiplicity of the corresponding knots to $n_{k}-1$, in which case the above condition simplifies to:

$$
\bar{V}_{i 1}=\bar{W}_{i} \text {, for } i=1 . . n_{u}
$$

Thus, fewer degrees of freedom are left for the least-squares fit, but a boundary is enforced. The above procedure applies to each of the four boundaries.

Compatibility between the boundary curves and the surface boundary curves must be ensured and can be accomplished by applying the Oslo algorithm. As suggested in [7], instead of uniform knot vectors, average knot vectors are computed so that more knots are allocated where there is a rapid change.

\section{EXAMPLE}

The implemented environment is: a Cyberware laser scanner; a Sony CCD camera; and a Silicon Graphics computer system and software.

The concrete example is based on a face mask model. Figure 5 (a)-(g) follows the different steps outlined in the previous section.

\section{CONCLUSIONS}

3D boundary detection of the 3D digitized points produced by laserbased scanners is problematic. This is due to the fact that the sensor data is often unreliable near sharp edges. With this paper we cope with the problem of surface reconstruction from 3D scattered data and 2D intensity image, while utilizing the surface boundary. The main stages of the proposed algorithm based on image processing, matching, parameterization and surface fitting technique. It seems that the reconstructed process was improved especially due to image processing and parameterization techniques: 1) Detecting the 3D boundary from which the base surface was dictated. 2) Parameterization that was calculated by projecting the $3 \mathrm{D}$ points on the sculptured base surface. The reconstructed surface according to the parameterization was used as a base surface for the next iteration. The feasibility of the method was illustrated by several examples mainly applied on complex sculptured surfaces.

As future work this method can be used as a base for 3D photography by integrating the CCD camera and the laser scanner technologies into one 3D camera. 


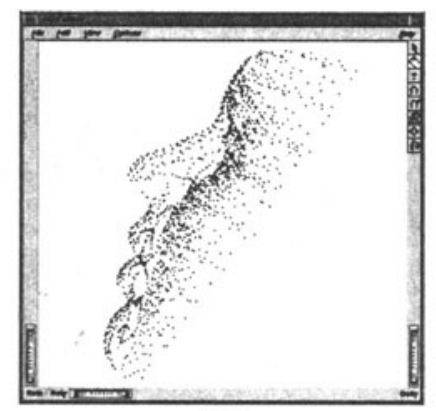

(a)

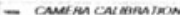

tere
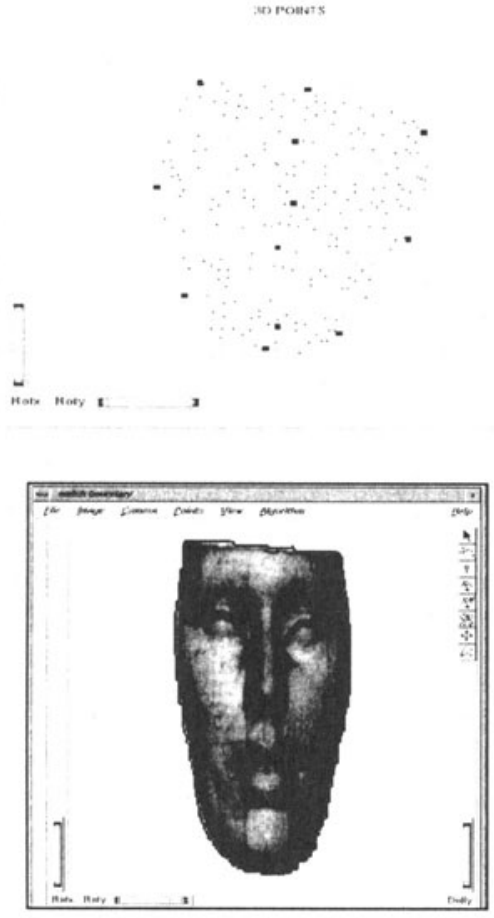

(d)

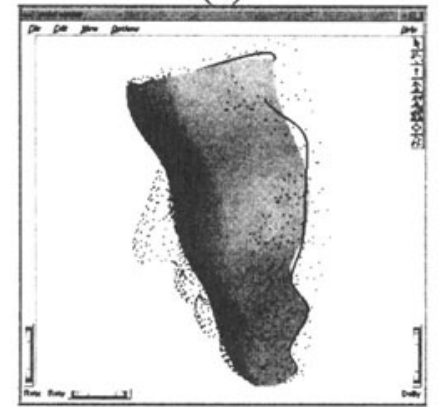

(f)

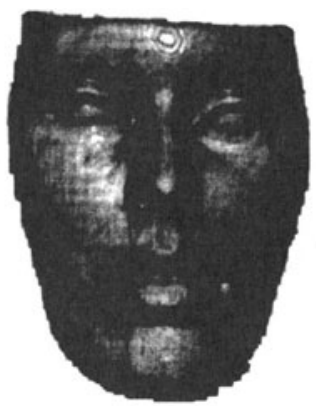

(b)

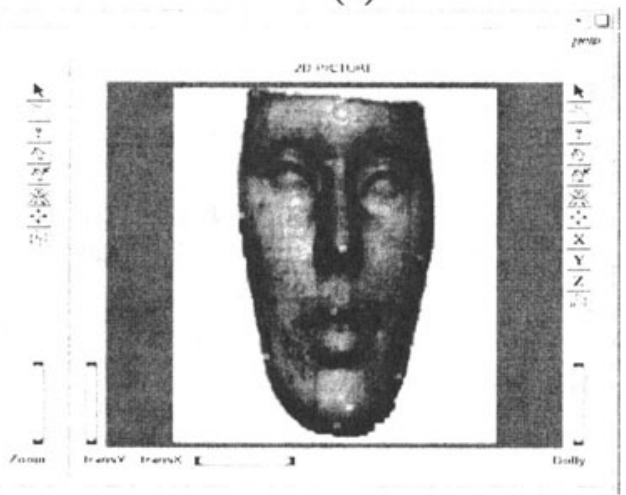

(c)

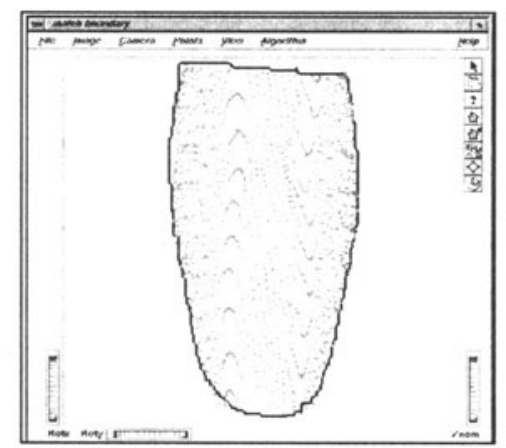

(e)

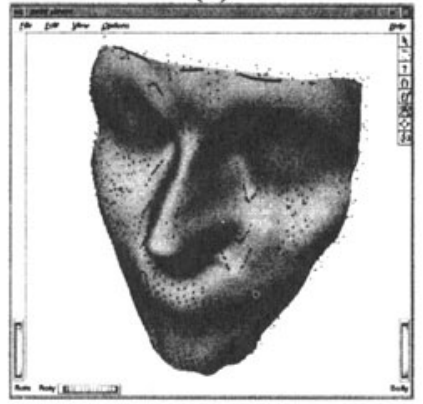

(g)

Figure 5: Steps in processing a mask object. 


\section{REFERENCES}

1. Bonitz P. and Krzystek P. Reverse Engineering in Combination with Digital Photogrammetry (ICEM SURF / ICEM PHOTO). In: Product Modeling for Computer Integrated Design and Manufacture, Chapman \& Hall, 1997.

2. Clark J. 3D Scanning Systems for Rapid Prototyping. Assembly Automation, vol. 17(3), 1997, pp. 206-210.

3. Faux, I.D. and Pratt, M.J. Computational Geometry for Design and Manufacture. Ellis Horwood 1981.

4. Fitzgibbon, A.W., Eggert, D., Fisher, R.B. High-Level CAD Model Acquisition From Range Images. Computer-Aided Design, vol. 29(4), 1997, pp. 321-330.

5. Herbert, M., Kweon, I., Kanade, T. 3-D Vision Techniques for Autonomous Vehicles. In: Vision and Navigation, The Carnegie Mellon Navlab. Kluwer Academic Publishers, 1990.

6. Hoschek, J., Lasser, D., Schumaker, L. (trans): Fundamentals of Computer Aided Geometric Design. A K Peters 1993.

7. Kruth, J.P. and Ma, W. Parameterization of Randomly Measured Points for Least Squares Fitting of B-spline Curves and Surfaces. Computer-Aided Design, 27(9), 1995, pp. 663-675.

8. Levin, M.D. Vision in Man and Machine. McGraw-Hill, 1985.

9. Mar, D. Vision. W.H. Freeman and Company, 1982.

10. Medioni, G. and Yasumoto Y. Corner Detection and Curve Representation Using Cubic B-Splines. Computer Vision, Graphics, and Image Processing, vol. 39, 1987, pp. 267 278.

11. Milroy, M.J., Weir, D.J., Bradley, C., Vickers, G.W. Reverse Engineering Employing a 3D Laser Scanner: A Case Study. Int. Journal of Advanced Manufacturing Technology, vol. 12(2), 1996, pp. 111-121.

12. Motavalli, S. and Bidanda, B. A Part Image Reconstruction System for Reverse Engineering of Design Modifications. Journal of Manufacturing Systems, vol. 10(5), 1991, pp. 383-395.

13. Park, H. and Kim, K. An Adaptive Method for Smooth Surface Approximation to Scattered 3D Points. Computer-Aided Design, vol. 27(12), 1995, pp. 929-939.

14. Piegl, L. and Tiller, W. The NURBS Book. Springer, 1995.

15. Seiler, A., Balendran, V., Sivayoganathan, K., Sackfield, A. Reverse Engineering from Uni-Directional CMM Scan Data. The Int. Journal of Advanced Manufacturing Technology, vol. 11(4), 1996, pp. 276-284.

16. Sinha, S.S. and Seneviratne, P. Single Valuedness, Parameterization and Approximating 3D Surfaces Using B-splines. In SPIE: Geometric Methods in Computer Vision II, 193204, 1993. 